\title{
Synergistic Antioxidant Activity between Honey and Phenolic Compounds ${ }^{+}$
}

\author{
Luminița Dimitriu 1,2, Daniel Preda ${ }^{1}$, Andra Nichițean ${ }^{2,3}$, Diana Constantinescu-Aruxandei 1,*, \\ Florin Oancea ${ }^{1,2, *}$ and Narcisa Băbeanu ${ }^{2}$ \\ 1 National Institute for Research \& Development in Chemistry and Petrochemistry - ICECHIM, \\ 060021 Bucharest, Romania; luminita.dimitriu@icechim.ro (L.D.); daniel.preda@icechim.ro (D.P.) \\ 2 Faculty of Biotechnologies, University of Agronomic Sciences and Veterinary Medicine of Bucharest, \\ 59 Mărăști Blvd., 011464 Bucharest, Romania; yourbesthoneysupplier@gmail.com (A.N.); \\ narcisa.babeanu@gmail.com (N.B.) \\ 3 Rom Honey Group Srl, 1 Grădinari Str., 051786 Iași, Romania \\ * Correspondence: diana.constantinescu@icechim.ro (D.C.-A.); florin.oancea@icechim.ro (F.O.) \\ + Presented at the 16th International Symposium "Priorities of Chemistry for a Sustainable Development" \\ PRIOCHEM, Bucharest, Romania, 28-30 October 2020.
}

Published: 9 November 2020

Keywords: honey; antioxidant activity; polyphenols; synergism

Honey is a natural product that has the characteristics of a deep eutectic natural solvent (NADES) due to the intermolecular interactions between monosaccharides and disaccharides, especially the hydrogen bonds formed between them. In previous research, we and others demonstrated that honey enriched in plant polyphenolic extracts presents synergic antioxidant effects. This work shows that specific polyphenolic compounds from plant extracts are involved and responsible for the synergic effects observed.

The content of phenolic acids from various plant materials (sea buckthorn, raspberry, propolis, tansy, and others) was analyzed by HPLC, and phenolic acids such as p-coumaric and ferulic acid were found in significant amounts. These phenolic compounds were chosen to test the putative synergy induced between phenolic acids and honey. Ferulic (FA) and p-coumaric (PA) acid were solubilized in $70 \%$ ethanol or honey at different concentrations. The antioxidant activity (AOA) of the samples was assayed using five spectrophotometric methods: radical scavenging activity (ABTS and DPPH) and reducing antioxidant power (CUPRAC, FRAP, and PFRAP).

The mixture between individual phenolic acids such as FA and PA, found in plant hydroalcoholic extracts, and honey presented a synergic increased antioxidant activity at all concentrations and for most of the antioxidant assays. No synergic effect was found between different polyphenols, indicating that the effect is the result of the interaction between individual polyphenols and honey.

Our study shows that phenolic acids, such as ferulic and p-coumaric acid, found in many plants, are involved in the synergic effect between plant extracts and honey. The synergic AOA between honey and the phenolic compounds may be due to the NADES characteristic of honey [1].

Acknowledgments: The work of this paper was supported by POC-A1-A1.2.3-G-2015-P_40-352-SECVENT, funded by cohesion funds of the European Union, in a subsidiary project 2236/2017 related to increasing polyphenols content into honey. 


\section{References}

1. Dai, Y.; Jin, R.; Verpoorte, R.; Lam, W.; Cheng, Y.C.; Xiao, Y.; Xu, J.; Zhang, L.; Qin, X.M.; Chen, S. Natural deep eutectic characteristics of honey improve the bioactivity and safety of traditional medicines. $J$. Ethnopharmacol. 2020, 250, 112460.

Publisher's Note: MDPI stays neutral with regard to jurisdictional claims in published maps and institutional affiliations.

(C) 2020 by the authors. Licensee MDPI, Basel, Switzerland. This article is an open access article distributed under the terms and conditions of the Creative Commons Attribution (CC BY) license (http://creativecommons.org/licenses/by/4.0/). 